\title{
EFFECTS OF PARATUBERCULOSIS ON PRODUCTIVITY IN DANISH DAIRY COWS
}

\author{
A. Kudahl ${ }^{1 *}$, S. S. Nielsen ${ }^{2}$ and J.T.Sørensen ${ }^{1}$ \\ ${ }^{1}$ Department of Animal Health and Welfare, Danish Institute of Agricultural Sciences, Foulum, Denmark. \\ ${ }^{2}$ Department of Animal Science and Animal Health, The Royal Veterinary and Agricultural University, \\ Copenhagen, Denmark.
}

Paratuberculosis is a chronic enteritis in dairy cattle and other ruminants caused by Mycobacterium avium subsp. paratuberculosis (MAP). Clinical outbreak causes economic losses due to reduced milk production and slaughter weight and susceptibility to other diseases, and at herd level involuntary culling and death rate are increased. During the last twenty years it has become more and more obvious, that also subclinical paratuberculosis may have an effect on milk production, fertility, milk quality, susceptibility to mastitis, slaughter weight and culling rate. However previous studies of these effects on productivity offer conflicting results, which may be due to herd specific circumstances. In this study antibodies to MAP in milk samples from 7994 cows in 108 Danish herds were tested with enzyme-linked immunosorbent assay (ELISA). Readings were recorded on a continuous scale as the optical density (OD). These OD values were standardised according to parity and stage of lactation as previous studies on the same material show significant increase in ELISA response with increasing parity and in the first and last weeks of the lactation of infected cows. In order to use the diagnostic information in recordings on a continuous scale, subdivision into a positive and negative group by choosing a cut-off value is avoided. Response variables expressing productivity are: milk yield, milk quality (fat, protein and somatic cell count), days open, slaughter quality and weight. At herd level effects on death and culling rate will be analysed. Besides the primary explanatory variable: adjusted ELISA response to MAP, covariables such as age at first calving, parity, calving season, housing system and herd size are included in the analysis. Effects on productivity will be analysed with multi-level regression analyses. A preliminary analysis comparing the lowest $10 \%$ with the highest $10 \%$ of the corrected OD-values indicate that average daily milk yield during the first 24 weeks of lactation, protein content of the milk, slaughter weight and quality are reduced in cows with high OD-values (probably infected cows) compared to cows with low OD-values (probably non-infected cows). More detailed analyses at cow and herd level will be presented at the 11th ICPD in August 2001. 